\title{
Research computer software technology auxiliary material dyeing process
}

\author{
Yan-li Hu
}

\begin{abstract}
In the context of large-scale industrial production, the traditional tie-dyeing process cannot accommodate large-scale mass production. The current representative computer software simulates the variation of the traditional tie-dye pattern into two methods: one is to re-group the pattern design by dividing the original pattern, and the other is to simulate the pattern of the tie-dye process by computer software. The former contributes to the rich pattern styles and colour matching changes, and the latter can provide a similar effect to tie-dyed textures, both to change the limitations of traditional handmade methods, and to achieve wholesale targets through the printing process. However, the tie-dye mode designed by electrical technology simulation is very stiff. Therefore, how to skillfully combine the traditional tie-dyeing process with computer software-assisted technology is the key to this paper.
\end{abstract}

Keywords: Computer design, Innovative patterns, Tie-dye process, Digital technology

\section{Introduction}

By virtue of the special artistic charm, traditional tie-dye has been widely disseminated worldwide and has farreaching impact. However, due to several constraints on handmade production, its development has been limited a lot caused by higher market price, fabrics tend to fade and larger unpredictability and other related problems. With the continuous development of science and technology, many computer software applications are released for combination and innovation of vivid and brand new technology and art according to the types of traditional processes and design arts. Different from initial making skills of traditional tie-dye process, digital art has successfully achieved the conversion of design materials from a traditional paper drawing into computer software application operated by designers, which create various types and kinds of process design works by the advantages of digital software and offer people an unprecedented new visual feast. Thus, in the breakdown of tie-dye art on the traditional process, the reference to digital art for modern tie-dye pattern design has a certain theoretical research value and strong practical application.

Tie-dyeing explains that "tie" is tied, tied, and tied; "dyeing" is dyeing. In the narrow sense, the traditional

Correspondence: 18079177621@163.com

Jiangxi Institute of Fashion Technology, No. 108, Lihu Middle Road,

Xiangtang Economic Development Zone, Nanchang, Jiangxi, Nanchang, Jiangxi, China

Springer Open dyeing and weaving process is a concept of combining designers with natural fibers and other fabrics [1]. A method of tying, sewing, screwing, clamping or selecting various manual folding methods forms a physical protection of the fabric. This anti-staining technology has also become a special artistic effect, showing a different texture pattern and colourful colour matching, which has a unique charm. When traditional tie-dye patterns are combined with computer software technology, new concepts emerge. It is different from the traditional dyeing and weaving process. It can use the high-tech and modern craftsmanship of modern dyeing and weaving techniques on any textile material. The aesthetic characteristics of traditional crafts reflect the aesthetic plane, three-dimensional or modern art. The artistic creation process of new graphics is produced by the blending of monochrome and multi-colour. In the information age, computer software technology uses digital processing technology as the main means, which provides a corresponding reference for modern tie-dyed art, and has become a new graphically important tool and main design language for contemporary designers.

Currently, the typical representatives are using digital software to simulate traditional tie-dye pattern changes, which could be operated by two methods, one is to re-group pattern design by splitting original patterns and another is to use Photoshop computer software to 
simulate the pattern of tie-dye process. The former is able to offer an effect similar to tie-dye texture, and the latter is helpful in rich style of patterns and changes of colour matching and can change the constraints of traditional handmade methods and achieve the wholesale target. However, the tie-dye pattern designed by electrical technology simulation is much stiffer. Therefore, how to combine traditional tie-dye process with digital technology much harmoniously is the key in this article [2].

\section{Trends of computer software designing patterns}

Tie-dye art has the charm of traditional culture. This is the value of our traditional art and crafts. We should make use of it to make traditional artistic features play a huge role in the modern design. The traditional tie-dyeing process utilizes purely hand-knotted knots to form a colour halo with different aesthetics, which make the final dyed fabric have a strong visual effect. It is a typical national symbol and cultural spirit. Traditional hand-dyed dyeing is done by hand-dying and dye with natural fabrics and natural dyes [3]. Although some chemical dyes have been added in modern times, there are many tie-dyeing processes which take a long time and require a skilled craftsmanship to obtain good tie-dyeing. The shortcomings of the pattern effect and low work efficiency also restrict the development of the traditional process.

Nowadays, with the rapid development of science and technology, computer software technology has helped tie-dye pattern-assisted design, and the digital technology of patterns has gradually become a new way of modern tie-dye design. Digital technology provides a great help for the pattern design and creativity for tie-dye art, fast and diverse acquisition of new patterns, Photoshop software with super-strong retouching skills and CoreLDRAW drawing software for drawing high-resolution vector graphics; these softwares help the designer's composition of the composition handy when creating. We can use a digital camera, scanner and other equipment to sort and collect the original material of the tie-dyed pattern. The tie-dyed pattern can be reorganized according to the materials collected in the computer and the new pattern design (as shown in Figs. 1 and 2). This greatly saves the time of traditional pattern making, and the designer can also use computer software to carry out rich pattern innovation in the design innovation of tie-dye art.

\section{Method: computer digital changes on traditional tie-dye process}

The natural texture beauty made by tie-dye process is the factor that people like and use such process; thus, digital-designed pattern will not focus on simulating tiedye pattern by using digital technology only but combine the effect of tie-dye process with digital technology; for breaking the single pattern and stylized presentation

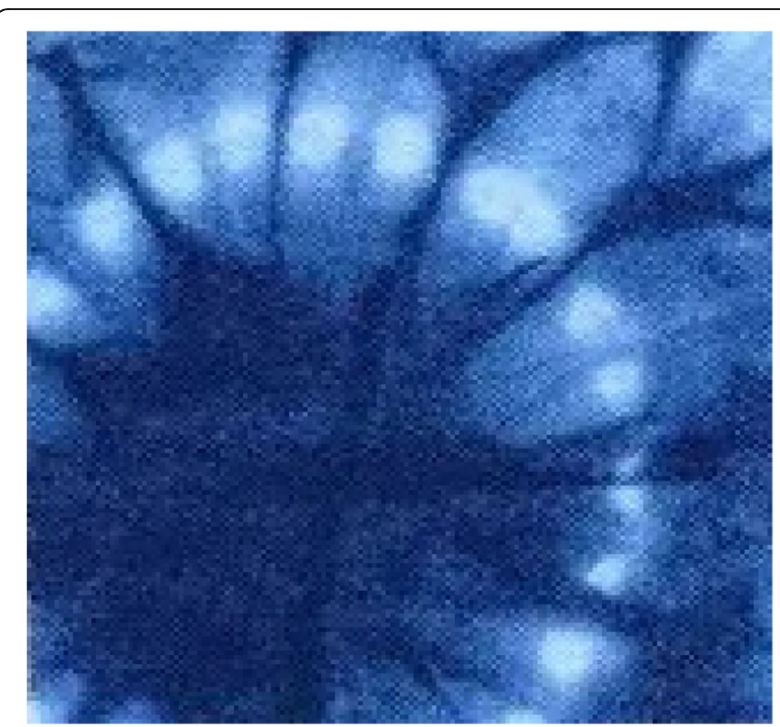

Fig. 1 Traditional flat seam tie-dyeing process [7]

techniques of handwork, the combining with modern emerging technologies has changed the creative way for tie-dye process design, which is exactly an innovative solution for digital tie-dye art.

Traditional handmade tie-dye patterns are diversified and contain some changing law if get arranged; the main elements constituting pattern change include "point", "line" and "face", which are the three basic elements. Such elements made by handmade tie-dye technologies will be scanned into computer software and will be applied with recombination and innovative design such as modification, arrangement, reproduction, permutation and combination

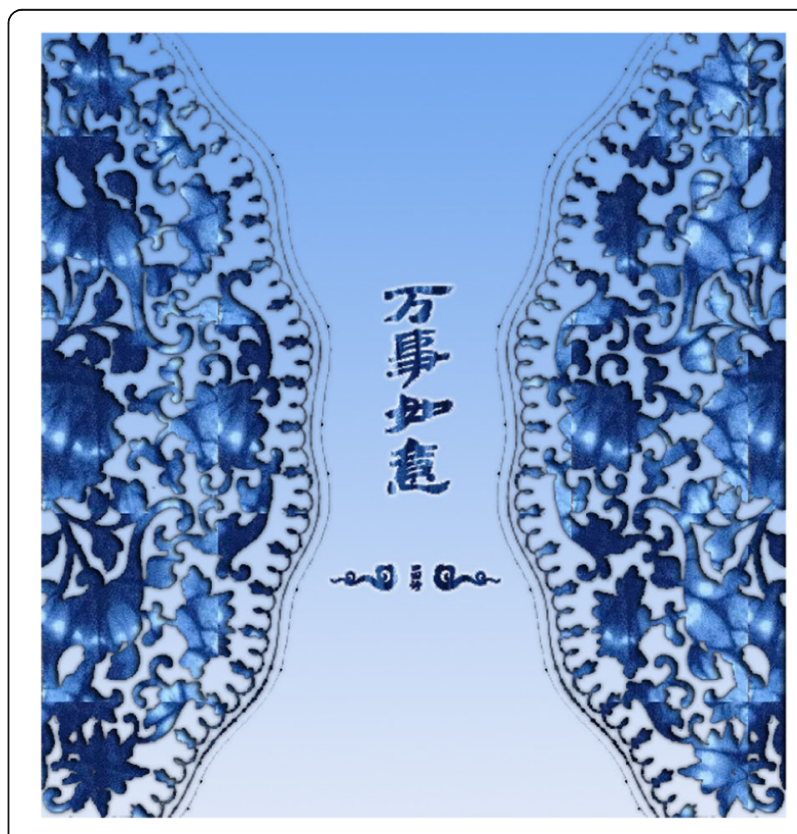

Fig. 2 Computer software technology reorganization tie-dye pattern [7] 
by using digital design methods. In this way, new patterns will be made by new unit pattern design, and batch production will be achieved by printing technology. Such technologies could not only retain the texture characteristics, natural and plain charm of traditional handmade tie-dye process, but also design patterns with rich styles by freely innovation. Moreover, the free matching of colour, position change, and simulation of fabric texture settled by digital technology change could promote the efficiency of manufacturing and production, as well as cut the cost.

We could find the tie-dye pattern innovation made by digital technologies from the view of point tie-dye pattern example: firstly, scan the traditional point-type tie-dye pattern into the computer and align the tone by tone scale; the unit element of point could be the combination of pattern on one or more pieces of tie-dye fabrics, then use CorelDraw/Photoshop software technology to get combination and permutation such as copy, collage or tie line by points, tie face by lines, form geometry and abstract shapes, which could avoid the perennial omission, error, colour omission and other mistakes during the application of the tie-dye handmade technology, be capable of repairing the marking made by combination and permutation through patch tool or stamp tool in PicTools, repairing operation could preferably retain the marking made by original patterns, which is the key to mix the spurious with the genuine (as shown in Figs. 3, 4, 5, 6 and 7).

In order to achieve the above-mentioned purpose, the article analyses the operation process of computer software, and the concrete steps of using the computer software technology to assist the change of the traditional material process are as follows:

S1-CoreLDRAW establishes the initial form of the image: open CoreLDRAW on the computer, create a new file in the Auto CoreLDRAW Classic mode, save it to the desktop, create a new file name 1.dwg and save 1.dwg flashback phenomenon in the operation process Can recover files;

S2-Process the image: operate within the 1.dwg file created in step S1 to create a desired line. Generally, tie-dyeing lines are circular arrays, hand-drawing or selecting a desired image, and forming the image into a rectangle. Array or circular array operation, scaling operation according to the demand through the offset, through the composition of the line to complete the desired pattern of the border;

S3-Initially save the image: adjust the image size of the border drawn by the pattern in step S2, mainly adjust the size and resolution of the image, and determine the reasonable parameters according to the size of the actual clothes. The higher the resolution, the

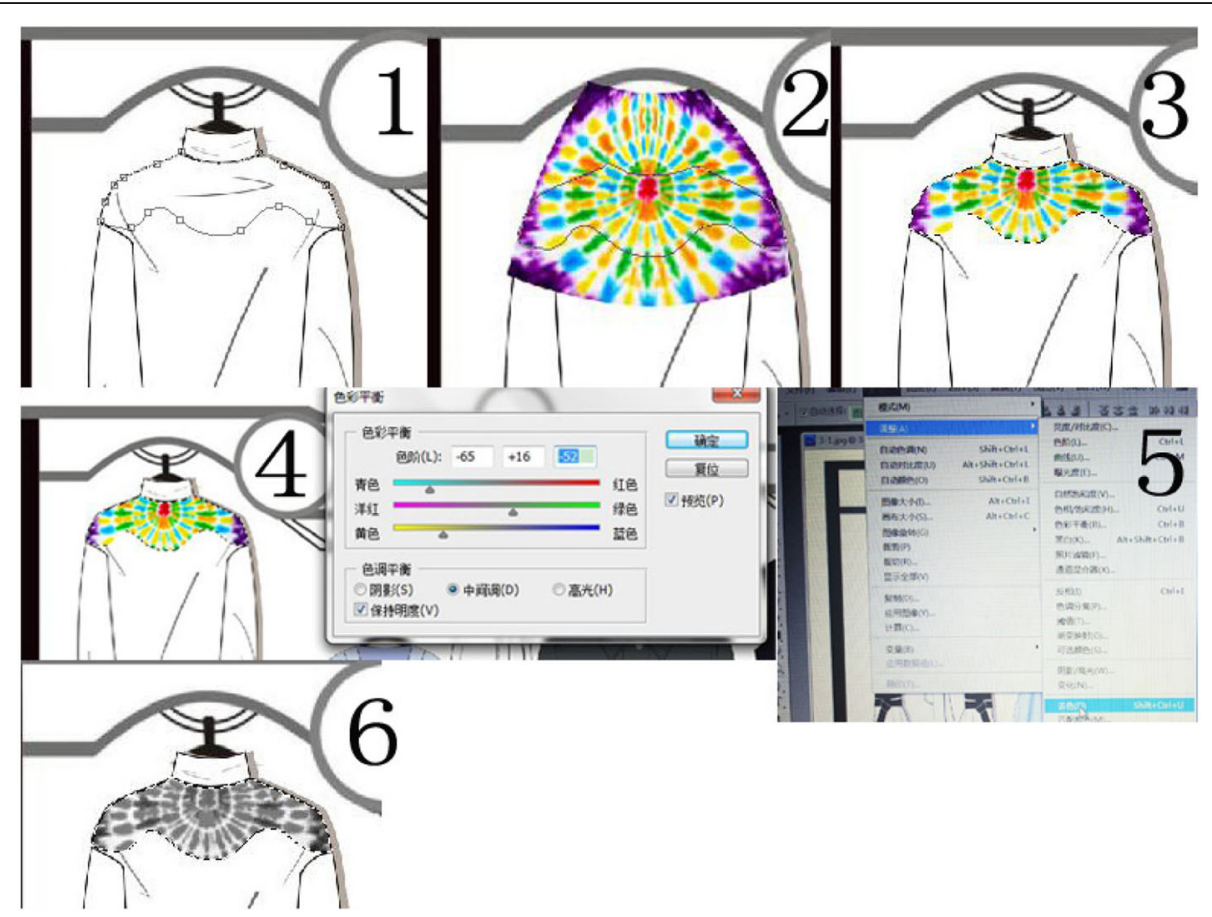

Fig. 3 Software operation process for recombining patterns based on the decomposition of original graphics. (The first kind of computer software technology is to recombine the pattern design according to the decomposition of the original graphics and to use the effect of forming a tie-dyed texture) [7] 


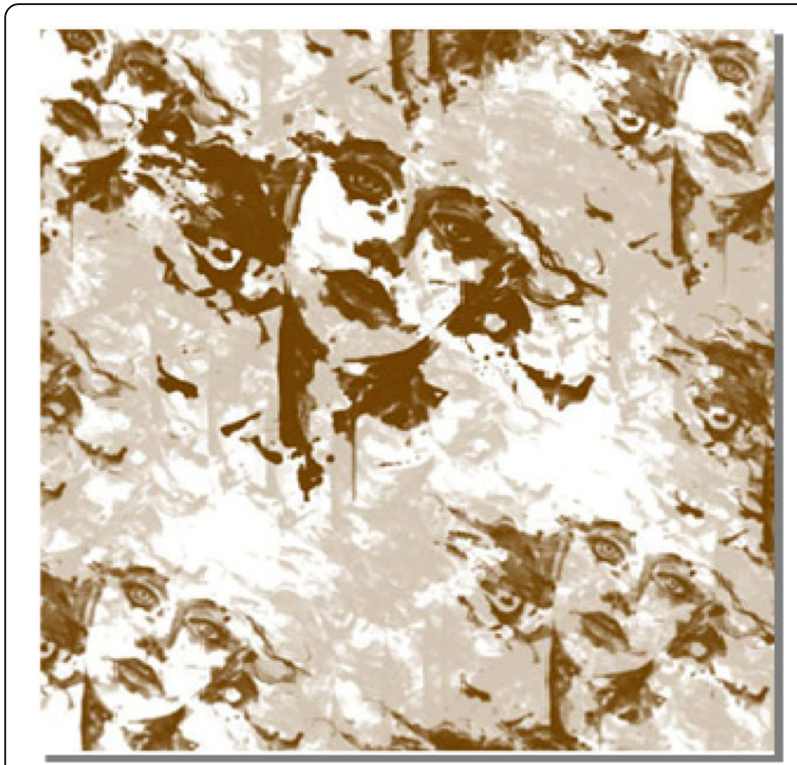

Fig. 4 Computer digital simulation tie-dye pattern. (The second is to use the Photoshop computer software painting method to imitate the tie-dye handmade pattern and use the effect of forming a tie-dyed texture) [7]

clearer the image. Save the image to 1.dwg; after saving, select all, adjust the line width and wide line width in the next step easier to operate at the corner, 1.dwg PDF file format export, save the name For Drawing1.pdf; S4-PS performs the image colour processing: open Adobe Photoshop, create and save to 1.psd, insert

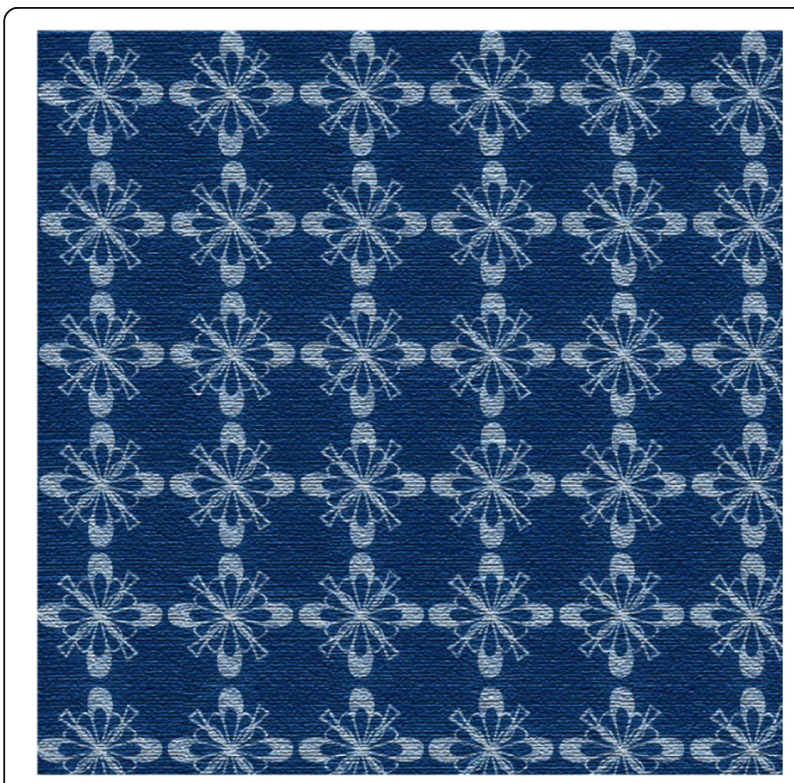

Fig. 5 Computer digital simulation tie-dye pattern. (The second is to use the Photoshop computer software painting method to imitate the tie-dye handmade pattern and use the effect of forming a tie-dyed texture) [7]

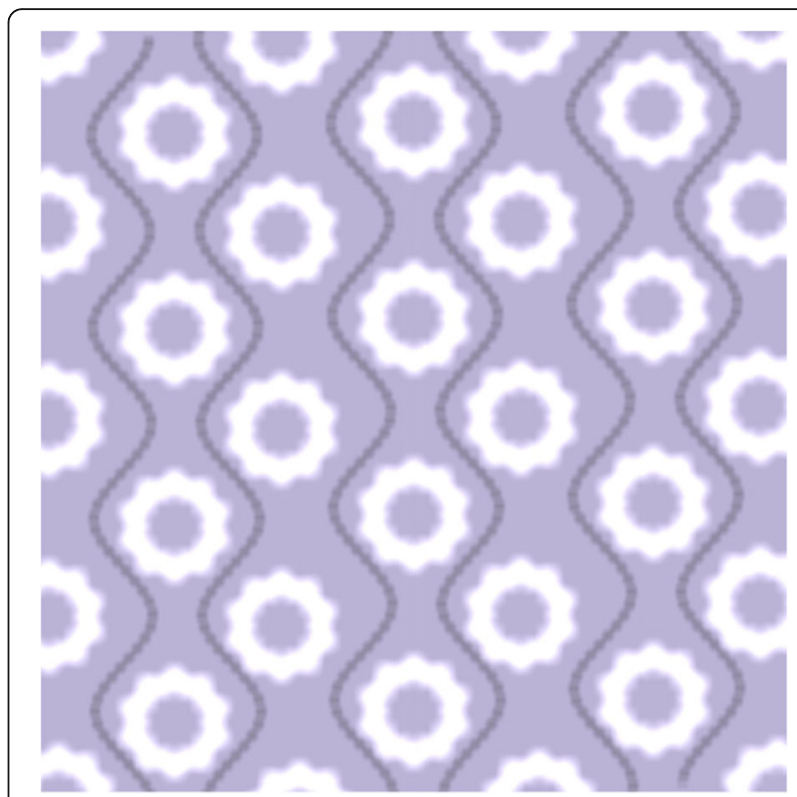

Fig. 6 Circle pattern (computer simulation tie-dye pattern). (The second is to use the Photoshop computer software painting method to imitate the tie-dye handmade pattern and use the effect of forming a tie-dyed texture) [7]

Drawing1.pdf printed in step S3 into Adobe Photoshop, add white coating to the inserted Drawing1.pdf file, and white The coating is at the bottom layer, and it is convenient to observe the file information of Drawing1.pdf, blur or erase the border of the existing pattern of Drawing1.pdf and perform the colour processing according to the desired target pattern. The colour processing includes the brightness of the target image, brightness, saturation, hue, etc.;

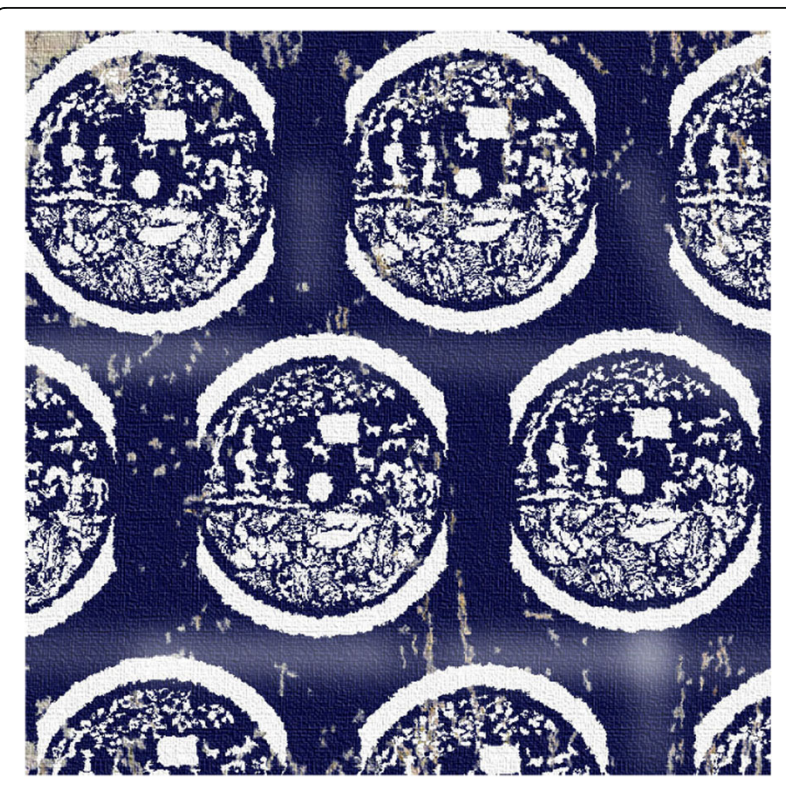

Fig. 7 Computer simulation tie-dye pattern [7] 
S5-Save the processed image: print the colour processed file in step S4 to a PDF file and save it to the desktop and save the file name as tie-dye, PDF; S6-Print to clothes: the computer connects to the hardware printing device and prints the pattern of tie-dye. PDF stored in step S5 to the clothes that need to be printed and dyed. The material of the clothes is cotton, hemp, wool, silk or chemical fiber, and the printing equipment is used. Digital transfer machine, printing and dyeing clothes in a constant room temperature of $120-140{ }^{\circ} \mathrm{C}$ for $3-5$ min fixed colour processing (as shown in Fig. 8).

On the left side of the figure is the finished product of the traditional tie-dyeing process; on the right is the finished product made with the combination of the digital pattern technology and the traditional tie-dyeing process, and the contrasting digital technology changes the colour of the self-contained, position swapping, fabric texture simulation and so on, but also improve the efficiency of production and reduce costs (as shown in Figs. 9, 10 and 11) [4].

The tie-dyed pattern can be reorganized according to the materials collected in the computer and the new pattern design (as shown in Figs. 9 and 10). These descriptions of the "bone and horseshoe" women's clothing let you watch chilling, using computer applications to transform the digital technology of the pattern into a new way of modern tie-dye design.

And the designer can also use computer software to carry out rich pattern innovation in the design innovation of tie-dye art.

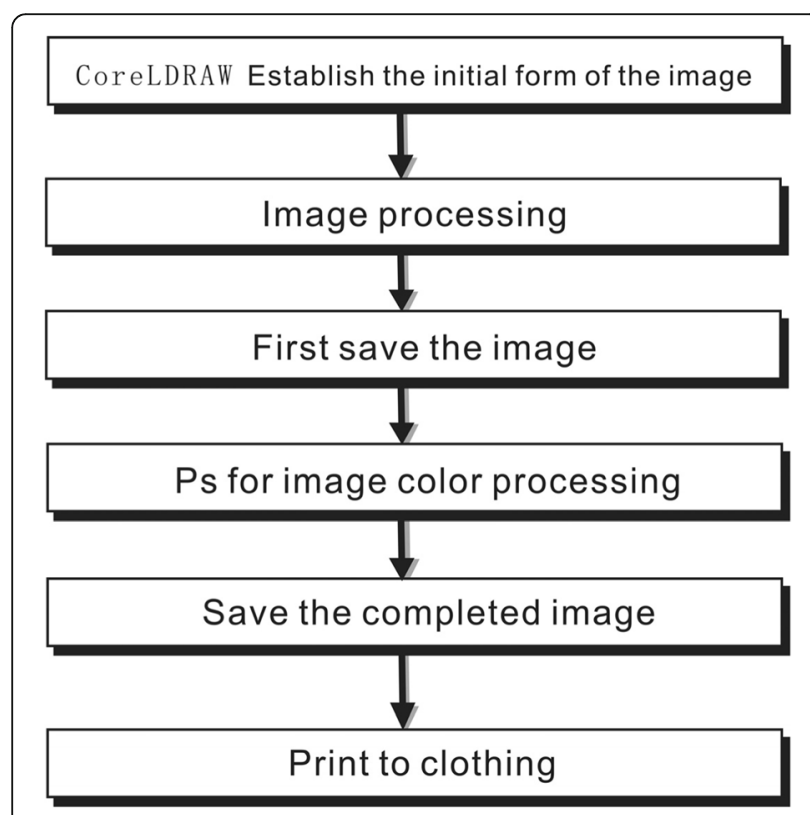

Fig. 8 Framework of the proposed method

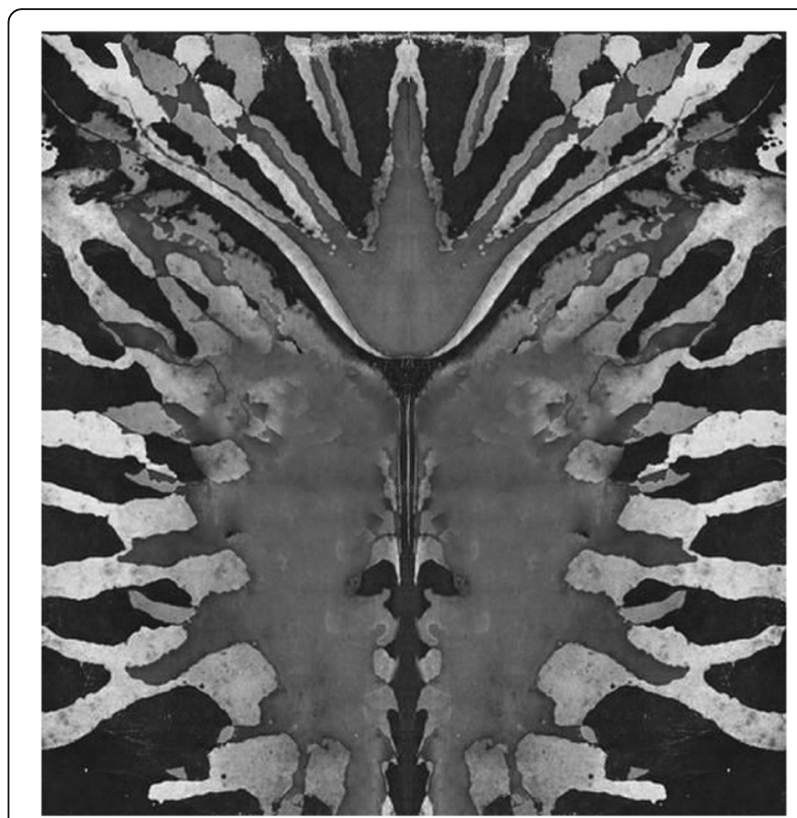

Fig. 9 Tie-dye process

\section{Results: the combination process of digital printing and traditional tie-dyeing of cashmere fabrics}

Digital printing of cashmere fabric with the combination of the traditional tie-dye process, this process will be digital printing with traditional tie-dye together effectively,

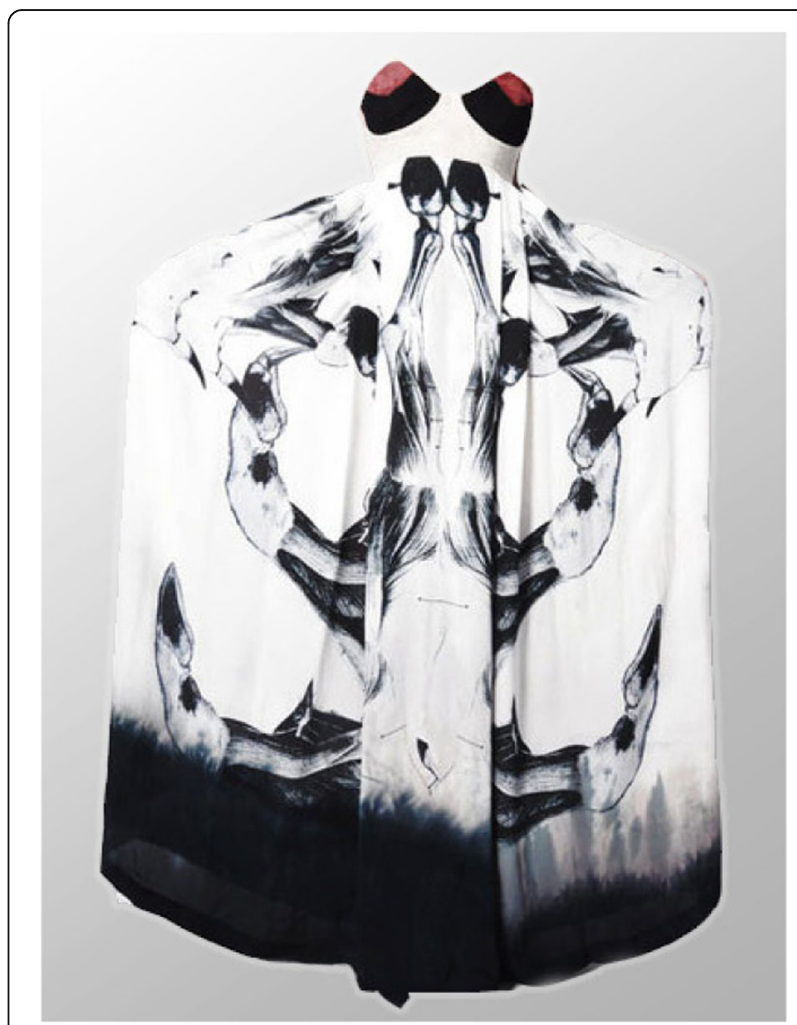

Fig. 10 Digitally rearranged pattern 


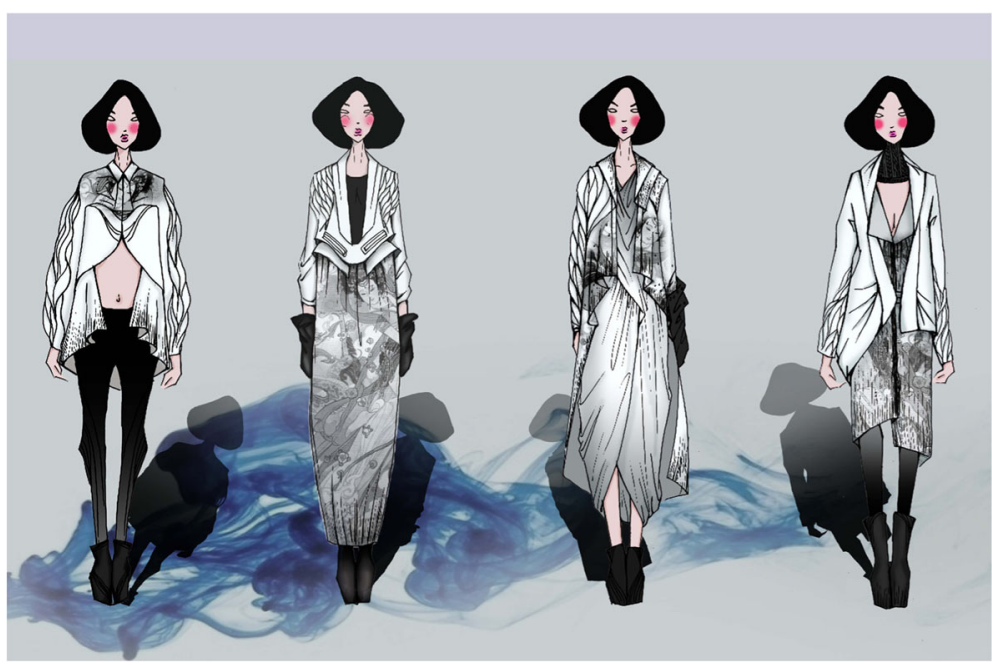

Fig. 11 Digitally rearranged pattern

both to preserve traditional tie-dye by hand skin texture characteristics and natural rustic charm, and can freely innovation design modelling is rich in design, improved the production efficiency, reduce the production cost, can continue to guarantee the quality of the dyed fabrics and artistic glamour. The invention discloses process of wool fabric after dyeing cashmere products can obviously improve the tinctorial yield which is low, and the wool fabric in the process of using easy to rub off phenomenon make the fabric after dyed presents design more fresh and vivid [5].

The following are the advantages of embodiments:

1. Will be a digital printing with traditional tie-dye effective together, through the computer software to the original traditional tie-dye graphics decomposition again combination pattern design, pattern design of combination again decomposition, and determined the tie-dye method, tie-dye colour and printing design, through this process, both to retain texture features of the traditional manual tie-dye and natural rustic charm, and can freely innovation design modelling is rich in design.

1. Using the digital technology change swap that deals with the independent collocation of colour, the position, fabric texture simulation and so on can improve the production efficiency, reduce production cost and continue to guarantee the quality of the dyed fabrics and artistic glamour.

2. Wool fabric dyeing process, the selection of solid colour aqueous solution under the condition of a specific process, can significantly improve the cashmere fabric after the dyed tinctorial yield is low and fixation rate is increased by $20 \sim 30 \%$, improve the utilization rate of the dye, tie-dye products after processing, the processing before the tonal and downy, feel is good, and not easy corrugate decolouring, completely solve the problem of serious rub off tie-dye products and make the fabric after being dyed presents design more fresh and vivid.

\section{Implementation of example 1:}

1. To establish a plan-the original traditional tie-dye is stored in the computer graphics for decomposition, then reassembled to complete the pattern design and pattern design of complete decomposition again and determine the tie-dye method, tie-dye colours and printing design;

2. Tie-dye-within the firm good cashmere cloth in the dye VAT dyeing, the dyeing process is: will dye at room temperature and water proportion ratio of dye solution formation, dye solution by heating rate of $2{ }^{\circ} \mathrm{C} /$ min up to $36^{\circ} \mathrm{C}$, add good cashmere fabric, immerse $35 \mathrm{~min}$, add soda ash and sodium sulfate, the amount of soda ash and sodium sulfate for the quality of the wool fabric was respectively $0.6 \%$ and $0.8 \%$; then rising at the rate of $1{ }^{\circ} \mathrm{C} / \mathrm{min}$ to $75{ }^{\circ} \mathrm{C}$, 40 min continuous heat preservation; finally, the cooling speed of $3{ }^{\circ} \mathrm{C} / \mathrm{min}$ temperature down to room temperature; the dyeing process is complete;

3. Fixation for the first time-tie-dye of cashmere fabrics and the fixation for the first time processing steps as follows: removal of twisted into a knotted line, will be dried after dyeing the cashmere fabrics until no water greater than $25 \%$ is present in the fabric. Under the condition of the steam temperature of $280^{\circ} \mathrm{C}$, cashmere fabric is steam air-dried for $16 \mathrm{~min}$, dried by dehydration, washed with water and dried by dehydration again. Soak the dehydration-dried wool fabric with a 
temperature of $15{ }^{\circ} \mathrm{C}$, the mass concentration of $30 \%$ solid colour in aqueous solution, soaking time for $15 \mathrm{~min}$, fixation described including salt, glacial acetic acid and aqueous solution of solutes dichloride melamine urea acid salt, the salt, glacial acetic acid and chloride different melamine urea acid salt quality ratio of 3:2:1.5. Dehydrate and dry, then wash with clean water, dehydrate again and dry again;

4. digital printing: digital printing of cashmere fabrics after solid colour processing is adopted according to the determined printing patterns;

5. 5. Solid colour-for the second time will finish the printing of cashmere fabric under $110{ }^{\circ} \mathrm{C}$ temperature, drying 2 min under the conditions of the second solid colour.

Implementation of example 2:

1. To establish a plan-the original traditional tie-dye is stored in the computer graphics for decomposition and then reassembled to complete the pattern design, pattern design of complete decomposition again and determined the tie-dye method, tie-dye colours and printing design;

2. Tie-dye-within the firm good cashmere cloth in the dye VAT dyeing, the dyeing process is: will dye at room temperature and water proportion ratio of dye solution formation, dye solution by heating rate of $2{ }^{\circ} \mathrm{C} / \mathrm{min}$ up to $40{ }^{\circ} \mathrm{C}$, add good cashmere fabric, soak for $30 \mathrm{~min}$, add soda ash and sodium sulfate, the amount of soda ash and sodium sulfate for the quality of the wool fabric was respectively $0.5 \%$ and $1 \%$; then rising at the rate of $1{ }^{\circ} \mathrm{C} / \mathrm{min}$ to $77^{\circ} \mathrm{C}$, 40 min of continuous heat preservation; finally, the cooling speed of $4{ }^{\circ} \mathrm{C} / \mathrm{min}$ temperature down to room temperature; the dyeing process is complete;

3. Fixation for the first time-tie-dyed of cashmere fabrics, the fixation for the first time processing, steps as follows: removal of twisted into a knotted line; after dyeing the cashmere fabrics, dry under $60{ }^{\circ} \mathrm{C}$ until no water more than $25 \%$ is present in the cashmere fabric. Under the condition of the steam temperature of $260{ }^{\circ} \mathrm{C}$, the wool fabric is steam-dried after $25 \mathrm{~min}$, dried by dehydration, washed with water and dried by dehydration again. Immerse dehydration drying of cashmere fabrics is $20{ }^{\circ} \mathrm{C}$, and the mass concentration of $20 \%$ solid colour in aqueous solution, soaking time is $20 \mathrm{~min}$. The solute of solid colour solution includes salt, glacial acetic acid and dichloroisocyanuric acid salt, among which, the quality ratio of salt, glacial acetic acid and dichloroisocyanurate is 5:1:1. Dehydrate and dry, then wash with clean water, dehydrate again and dry again;
4. Digital printing is adopted for cashmere fabrics after solid colour processing according to the determined printing patterns;

5. Solid colour-for the second time will finish the printing of cashmere fabric under a temperature of $80{ }^{\circ} \mathrm{C}$, drying 3 min under the conditions of the second solid colour.

Implementation of example 3:

1. To establish a plan-the original traditional tie-dye is stored in the computer graphics for decomposition, and then reassembled to complete the pattern design, pattern design of complete decomposition again, and determined the tie-dye method, tie-dye colours and printing design;

2. Tie-dye-within the firm good cashmere cloth in the dye VAT dyeing, the dyeing process is: will dye at room temperature and water proportion ratio of dye solution formation, dye solution by heating rate of $2{ }^{\circ} \mathrm{C} / \mathrm{min}$ up to $38^{\circ} \mathrm{C}$, add a good cashmere fabric, immerse the $32 \mathrm{~min}$, add soda ash and sodium sulfate, the amount of soda ash and sodium sulfate for the quality of the wool fabric was respectively $0.4 \%$ and $1.2 \%$; Then rising at the rate of $1{ }^{\circ} \mathrm{C} / \mathrm{min}$ to $75^{\circ} \mathrm{C}, 40 \mathrm{~min}$ continuous heat preservation. Finally, the cooling speed of $5{ }^{\circ} \mathrm{C} / \mathrm{min}$ temperature down to room temperature; the dyeing process is complete;

3. Fixation for the first time-tie-dyed of cashmere fabrics, the fixation for the first time processing, steps as follows: removal of twisted into a knotted line, after dying the cashmere fabrics, dry under $55^{\circ} \mathrm{C}$ until no water more than $25 \%$ is present in the cashmere fabric. Under the condition of the steam temperature of $270{ }^{\circ} \mathrm{C}$, the wool fabric is steam-dried for $20 \mathrm{~min}$, dried by dehydration, washed with water and dried by dehydration again. Immerse dehydration drying of wool fabric is $18{ }^{\circ} \mathrm{C}$, the mass concentration of $25 \%$ solid colour in aqueous solution, soaking time is $18 \mathrm{~min}$. The solute of solid colour solution includes salt, glacial acetic acid and dichloroisocyanuric acid salt, among which, the quality ratio of salt, glacial acetic acid and dichloroisocyanurate is 4:1. Dehydrate and dry, then wash with clean water, dehydrate again and dry again;

4. Digital printing is adopted for cashmere fabrics after solid colour processing according to the determined printing patterns;

5. Solid colour-for the second time will finish the printing of cashmere fabric under a temperature of $90{ }^{\circ} \mathrm{C}$, drying $2.5 \mathrm{~min}$ under the conditions of the second solid colour. 
The tests were conducted on the soaping fastness of cashmere products made from $1 \sim 3$, and the results were shown in Table 1.

After testing, all cashmere products made from 1 3 are in accordance with the test standard of $\mathrm{GB} / \mathrm{T}$ 3921-2008 textile colour fastness test.

It is proved by the embodiment that it can preserve the texture features of traditional manual tie-dye and the natural and simple charm and can creatively design rich patterns. Fixation of wool fabric was increased by $20 \sim 30 \%$, improve the utilization rate of the dye and cashmere products, made by the processing before the feel is good, and not easy corrugate rub off, presents the design of more fresh and vivid.

\section{Discussions: significance on the integration of modern tie-dye with digital art}

The advantage of computer-aided design in the creation of tie-dye patterns is an important means of modern design on modern dyed fabrics. Its role cannot be replaced by other design methods, such as highly mature technology, widely used in clothing computer-aided design systems., printing-aided design system and intelligent pattern design system. Therefore, for the modern digital art, the superiority of digital art and the human-computer interaction interface friendly human-computer interaction interface design mode has a good market application prospect.

Firstly, from the view of the operation method, electronic pattern design made by human-machine cooperation reflects the intelligence of electronic technology in traditional processes. The drawing made by the computer software could promote the quality and efficiency of pattern design. The modern tie-dye design incorporating the digital art design has the advantages of high quality and richness of the traditional tie-dyed design and the strong advantages of serialization, which can make international buyers keep up with fashion trends.

Secondly, The traditional technology combined with the computer software number technology realizes the working state of man-machine combination. Using the free combination ability of computer software technology in pattern creation, it is also inevitable to use the computer technology to form a new discipline cross-type creation method, which is difficult to draw out the texture pattern and accidental pattern. The modern tie-dye pattern design

Table 1 Tests were conducted on the soaping fastness of cashmere products

\begin{tabular}{lll}
\hline The sample & \multicolumn{2}{l}{ Soaping fastness (level) } \\
\cline { 2 - 3 } & On colour fastness & Fade in colour fastness \\
\cline { 2 - 3 } Implementation example 1 & 5 & $4 \sim 5$ \\
Implementation example 2 & 5 & $4 \sim 5$ \\
Implementation example 3 & 5 & 4 \\
\hline
\end{tabular}

integrated with computer software technology reflects the new means of "human-machine merger" design. The design tools using computer software technology encourage the designers to actively accept the highefficiency sensory impact of computer materials, which can continuously stimulate and guide designers design creative potential and creative inspiration. Simultaneously, the strong storage function is a huge advantage of computer software design, which could be used to establish a database with rich and variable tie-dye pattern resources. Computer means could significantly reduce the time for production and sampling and substantially cut the cost of sampling due to the reduction of the technology process, which must bring enterprises with more market opportunities.

At last, from the perspective of artistic effects, modern tie-dye patterns are a variety of styles, creative and dynamic multi-dimensional pattern textures. It breaks through the traditional single tie-dye pattern, maximizing the creativity of its graphics and satisfying the requirements of the times and the call of the personalized market. The computer software technology is used to carry out the secondary design of the tie-dye pattern, which not only breaks through the simple colour smudge effect of the traditional craftsmanship but also presents a new and modern abstract art sense. And the appropriate use of computer graphic art design on the background of this large "face" will make the tie-dyed products more artistic and meet the needs of tie-dyed textile and apparel design, and more adapt to the consumption habits of modern people.

The computer software technology revolution not only brings people a modern aesthetic feeling of "postdesign", but also incorporates the styles of modern digital technology. The styles are diverse, and the various popular factors and different categories of fashion are better. The performance characteristics of art are flexibly and freely applied to the performance of contemporary art design. At the same time, it also tells us that the combination of abstract and concrete graphic elements and popular colour harmony has become a new form of appreciation, as well as the modern tie-dye design method and unique style of artistic creation.

\section{Conclusions}

In short, the combination of computer technology and creative modern graphic design is an indispensable expression of modern pattern dyeing design techniques. The advantages of computer assistant design with digital art on pattern creation are important means for modern pattern design on modern dyed fabrics, and such function will never be replaced by any other design means. Thus, for modern digital process technologies, high-tech means could not only change the traditional mode and production level of the pattern process but also breakthrough the expression 
form of pattern creation. How to blend traditional tie-dye pattern process into lifestyles and forms today, make innovation suitable for demands in the era of technology development market and make natural digital dyeing, which is more suitable for consumer function and aesthetic requirements, become the fashion trend and target in future and have good market prospects. Better serve all areas of process design [6]. Just like the classic cowboy culture art, he also uses the structural craft design to break through the traditional craftsmanship to obtain new visual effects and craftsmanship. Known as the "art dyeing and finishing" modern dye process. In the modern culture, craftsmanship and artistic techniques, they are all involved with the help of modern high-tech technology. Therefore, they have been able to break through the traditional classics in the aspects of craftsmanship, dyeing and finishing technology, graphic design and colour matching. Gain a new level of innovation to create a new art of pattern craftsmanship. It is accommodated by the aesthetic and culture of the times.

\section{Abbreviations}

CAD: Computer-aided design; PDF: Portable Document Format; RIP: Raster image processor

\section{Acknowledgements}

The author thanks the editor and anonymous reviewers for their helpful comments and valuable suggestions.

\section{Funding}

Fund project: 2016 Jiangxi Provincial Science And Technology Department Scientific Research Project "modern digital pattern technology innovation traditional tie-dye craft research" stage result, project number: GJJ161204.

Availability of data and materials

Request from the author.

\section{Author's contributions}

The author has completed the discussion of the work described in this article. The author completed his dissertation independently. In the version of this paper, author YH showcases the practice case of the paper and showered different versions of the paper. The author read and approved the final manuscript.

\section{Author's information}

Jiangxi province, Jiangxi Institute of Fashion Technology, engineering master, associate professor, No. 108, Lihu Middle Road, Xiangtang Economic Development Zone, Nanchang, Jiangxi, China, 330201.

Hu Yan-li has a Bachelor's Degree in Fine Arts from Jiangxi Normal University, China, 2014; Master's Degree in Industrial Design Engineering, Qingdao University. In 1998, she joined the Jiangxi Institute of Fashion Technology as a teacher. In 2015, she got the title of associate professor of fashion design. She joined the association of Chinese Fashion Designers Association in 2004. In 2010, she started to create a computer technology practice studio with her colleagues, combined with the school's dyeing and weaving studio, to study the traditional image changes with software technology.

Ethics approval and consent to participate Approved.

Consent for publication Approved.

\section{Competing interests}

The author declares that she has no competing interests and declares that the work described is an original study that has not been published before and is not considered elsewhere, in whole or in part.

\section{Publisher's Note}

Springer Nature remains neutral with regard to jurisdictional claims in published maps and institutional affiliations.

Received: 4 June 2018 Accepted: 2 August 2018

Published online: 05 September 2018

References

1. G Chen, Research on the innovative design of traditional tie-dye craft and modern digital technology. Art Technol. 06 (2016) 427

2. J Yao, Application of computer aided design in the teaching of tie-dye craft. Text. Ind. Technol. 03 (2017) 69-70

3. C Yang, Study on tie-dye craft and its artistry. Dyeing Finish. Technol. 11 (2016) $6-8+13$

4. Z Liu, B Xu, X Su, X Liu, C Xie, Application of digital printing in garment fabric creation. Shanghai Text. Sci. Technol. 04 (2013) 46-47+50

5. T Yan, Y Bai, Z Zhang, Digital inkjet printing of cashmere fabrics. Print. Dyeing 17 (2006) 16-18

6. X Hu, Brief analysis on the application of modern tie-dye craft in clothing design. Technol. Inf. 02 (2014) 173

7. S Li, Computer technology simulation of traditional printing and dyeing pattern design. Beijing Institute of Fashion. 68 (2015)

\section{Submit your manuscript to a SpringerOpen ${ }^{\circ}$ journal and benefit from:}

- Convenient online submission

- Rigorous peer review

- Open access: articles freely available online

- High visibility within the field

- Retaining the copyright to your article

Submit your next manuscript at $\boldsymbol{\nabla}$ springeropen.com 